\section{Altersbezüge erst ab 67 - das geht}

Die Anhebung der Regelaltersgrenze seitens ärztlicher Versorgungswerke von 65 auf 67 Jahre ist rechtens. Wie das Oberverwaltungsgericht Koblenz entschieden hat, müssen nicht nur Angestellte, sondern auch Freiberufler wie Ärzte oder Rechtsanwälte länger arbeiten (6 C 11098/11).

Wegen der gestiegenen Lebenserwartung wird das Rentenalter in der gesetzlichen Rentenversicherung derzeit in Stufen von 65 auf 67 Jahre angehoben. Nach Angaben der Arbeitsgemeinschaft Berufsständischer Versorgungseinrichtungen (ABV) in Berlin liegt die Lebenserwartung der Mitglieder der Versorgungswerke sogar vier Jahre über dem Bevölkerungsdurchschnitt. mwo

\section{Einnahme-Überschuss-Rech- nung: Formular bleibt Pflicht}

Ärzte und andere Freiberufler müssen ihre Einnahmen und Ausgaben dem Finanzamt weiterhin nach einem vorgeschriebenen Vordruck, der „Anlage EÜR", aufschlüsseln. Dies hat der Bundesfinanzhof (BFH) in München jetzt in einem Urteil bestätigt (X R 18/09). Dabei ermitteln die Praxen wie gewohnt ihren Gewinn nach der Einnahmen-Überschuss-Rechnung (EÜR) denn Freiberufler und Kleinbetriebe sind von der Bilanzierungspflicht befreit. Sie müssen die Daten anschließend aber für das Finanzamt in die „Anlage EÜR“ übertragen. maw

\section{Fachgrenzen gelten auch in einer Gemeinschaftspraxis}

Auch innerhalb einer Gemeinschaftspraxis muss die Trennung zwischen haus- und fachärztlicher Versorgung eingehalten werden. Das geht aus einem Urteil des Bundessozialgerichts (BSG) in Kassel hervor (B 6 KA 6/11 R). In einer internistischen Gemeinschaftspraxis hatte ein Hausarzt seinen Kollegen, der einen fachärztlichen Schwerpunkt als Gastroenterologe hat, im Urlaub vertreten und eine fachärztliche Zusatzpauschale abgerechnet. Zu Unrecht, so das BSG, da die Fachgrenzen auch in einer Gemeinschaftspraxis Gültigkeit hätten.

\section{Praxiswert: Einmischung der Zulassungs- ausschüsse nicht erwünscht}

Wenn sich die beteiligten Ärzte über den Wert einer Praxis einig sind,
dürfen die Zulassungsgremien nach einer Entscheidung des
Bundessozialgerichts nicht noch selbst einen Praxiswert ermitteln. $\mathrm{m}$ Streitfall hatte eine Psychotherapeutin in Baden-Württemberg sich mit allen an einer Übernahme ihrer Praxis interessierten Kollegen auf einen Verkehrswert von 40.000 Euro geeinigt. Trotzdem holte der Berufungsausschuss ein eigenes Gutachten ein, das einen Wert von 35.000 Euro ergab. Auch das gefiel dem Ausschuss nicht. Ein weiteres Gutachten ergab daraufhin einen immateriellen Praxiswert von Null. So verblieb nur noch ein Sachwert von 2.940 Euro. Das Landessozialgericht Baden-Württemberg monierte zwar, der Ausschuss habe den Widerspruch der beiden Gutachten nicht aufgeklärt. Bundesweit sorgten die Stuttgarter Richter aber für Aufsehen mit ihrer Aussage, der Ausschuss sei grundsätzlich berechtigt gewesen, einen Praxiswert festzusetzen (L 5 KA 1323/09).

Dem trat das Bundessozialgericht nun klar entgegen: Wenn sich der abgebende Arzt mit einem oder auch mit mehreren Interessenten auf einen Praxiswert einigen kann, haben sich die Zulassungsgremien nicht mehr einzumischen (B 6 KA 39/10 R). Ärzte haben damit die Möglichkeit, zeitraubende Gutachterverfahren zu umgehen und Nachfolgeentscheidungen zu beschleunigen. Nur wenn es verschieden hohe Gebote für die Praxis gibt, müssen nach dem Kasseler Urteil die Zulassungsgremien einen Wert festsetzen. Dieser darf aber nicht unter dem niedrigsten Gebot liegen, weil sich die Interessenten zumindest in dieser Höhe einig sind. mwo
Abrechnungstipp!

\section{Auch beim Schlaganfall ist Prävention Trumpf}

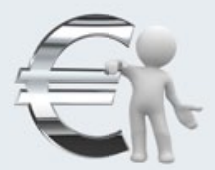

- Der Wunsch der Patienten nach Gefährdungsanalyse in punkto Schlaganfall wird häufiger. Speziell beim Neurologen bietet sich hier ein Untersuchungsprofil an, das als IGeL liquidiert werden kann, vorausgesetzt der Patient ist symptomfrei.

Neben einer ausführlichen Beratung (Nr. 1; Nr. 3 nur bei zeitlich getrennter Leistung) und klinischen Untersuchung (Nrn. 7 und 800) kommen doppler-sonografische Untersuchungen zur Anwendung. Daneben können gegebenenfalls zur Komplettierung der Hirnfunktionen ein EEG oder aber auch eine orientierende Testuntersuchung nach Nr. 857 erbracht werden. Die Nr. 857 ist dabei insgesamt nur einmal pro Sitzung abrechenbar, auch bei mehreren

\begin{tabular}{|c|c|c|}
\hline & & Tabelle 1 \\
\hline $\begin{array}{c}\text { GOÄ- } \\
\text { Nr. }\end{array}$ & Leistung & $\begin{array}{c}\text { Betrag } \\
1,15-/ 1,8 \\
/ 2,3-\text { fach }\end{array}$ \\
\hline 1 & Beratung & $10,72 €$ \\
\hline 3 & Ausführliche Beratung & $20,11 €$ \\
\hline 7 & Untersuchung Thorax, Carotiden & $21,45 €$ \\
\hline 800 & Neurologische Untersuchung & $26,14 €$ \\
\hline 801 & Psychiatrische Untersuchung & $33,52 €$ \\
\hline 857 & Orientierende Testuntersuchung & $12,17 €$ \\
\hline 645 & Doppler hirnversorgende Arterien & $68,20 €$ \\
\hline 827 & EEG & $81,11 €$ \\
\hline
\end{tabular}
Testuntersuchungen. In diesem Fall kann man auf einen erhöhten Multiplikator bis 3,5 ausweichen, was dann einem Honorar von 23,66€ entspräche. Fazit: Diese Vorsorgeuntersuchung etwa nach den Nrn. 1-800-645-857 generiert ein Honorar von 117,23 €; hierbei könnte dann eine Pauschale von $100,00 €$ oder $110,00 €$ vereinbart werden - mit entsprechender Wahl der Multiplikatoren auf dem Behandlungsvertrag mit Kostenvoranschlag und auf der Rechnung. Dr. med. Heiner Pasch 